\title{
Effect of the GoKnee Intervention on Knee Range of Motion, Pain, and Functional Mobility after Total Knee Arthroplasty
}

\author{
Suzanne Greenwalt ${ }^{1,2}$, Lauren Addison ${ }^{3}$, Kylene Bauer ${ }^{4}$, \\ Connor Bresnahan', Emma Oothout ${ }^{6}$, Amy Poynter ${ }^{7}$ \\ ${ }^{1}$ Associate Professor of Physical Therapy Belmont University, Nashville, TN, USA \\ ${ }^{2}$ Adjunct Faculty Department of Physical Therapy, Arizona School of Health Sciences, \\ A.T. Still University, Mesa, AZ, USA \\ ${ }^{3}$ BenchMark Physical Therapy, Lewisburg, TN, USA \\ ${ }^{4}$ AW Health Care, St. Louis, MO, USA \\ ${ }^{5}$ Indiana Physical Therapy, Warsaw, IN, USA \\ ${ }^{6}$ TriStar Centennial Medical Center, Nashville, USA \\ ${ }^{7}$ Body One Physical Therapy, Fishers, IN, USA \\ Email: suzanne.greenwalt@belmont.edu,sgreenwalt@atsu.edu, lauren.addison94@yahoo.com,kylenebauer@gmail.com, \\ Cbreshnahan@indianapt.com,emma.oothout@pop.belmont.edu, amy.poynter@aol.com
}

How to cite this paper: Greenwalt, S., Addison, L., Bauer, K., Bresnahan, C., Oothout, E. and Poynter, A. (2021) Effect of the GoKnee Intervention on Knee Range of Motion, Pain, and Functional Mobility after Total Knee Arthroplasty. Open Access Library Journal, 8: e7999.

https://doi.org/10.4236/oalib.1107999

Received: September 24, 2021

Accepted: October 23, 2021

Published: October 26, 2021

Copyright () 2021 by author(s) and Open Access Library Inc.

This work is licensed under the Creative Commons Attribution International License (CC BY 4.0).

http://creativecommons.org/licenses/by/4.0/ (c) (i) Open Access

\begin{abstract}
The prevalence of total knee arthroplasties (TKA) is rapidly increasing, but there is currently no standardized postoperative treatment approach, or a single treatment shown to be significantly better than others. The GoKnee device was created by physical therapists as an affordable treatment option after TKA to help patients achieve faster recovery of function. The purpose of the current study was to investigate whether the GoKnee device significantly improved objective and functional measurements after TKA. Twenty-nine patients who had recently undergone TKA participated. All data were collected by licensed physical therapists in a skilled nursing facility or home health care setting. In addition to a traditional TKA rehabilitation program, participants also followed the GoKnee protocol, which addresses their knee flexion range of motion, knee extension range of motion, and quadriceps strengthening when they use the device. Using a repeated measures design, the outcome measures of pain, knee flexion, knee extension, Timed Up and Go score, gait velocity, and 30-second sit-to-stand score were recorded at various times during treatment sessions. Differences $(P<0.001)$ were found for all variables for postoperative days 2 - 6 to 7 - 11. Improvements were also found for pain $(P=0.008)$ and knee flexion $(P=0.001)$ for postoperative days 7 - 11 to 12 - 16. No other differences were found (all $P>0.012$ ). Results sug-
\end{abstract}


gested that postoperative TKA patients had statistically significant improvements in functional and objective outcome measures after using the GoKnee device. However, with no control group for comparison, the GoKnee device should be used with caution until additional research is conducted.

\section{Subject Areas}

Kinesiology, Nursing

\section{Keywords}

GoKnee, TKA, TKA Rehabilitation, Total Knee Arthroplasty

\section{Introduction}

Total knee arthroplasties (TKA) are projected to increase to 3.48 million performed per year by 2030 [1]. Despite this increasing prevalence, many patients are unable to return to previous levels of function after the procedure. Further, patients are commonly discharged from physical therapy services with functional deficits still present. When compared with healthy older adults, Bade et al. [2] found that participants still had significant deficits six months after TKA in quadriceps strength, knee range of motion (ROM), stair climbing speed, Timed Up and Go (TUG) time, and six-minute walk test time.

Despite the growth in volume and evolution of surgical techniques, rehabilitation for postoperative TKA patients has remained consistent for many years. In general, patients complete a progressive program that includes exercises to increase strength and ROM, proprioceptive exercises, and functional training [2] [3] [4]. Additional interventions include slide board exercises and continuous passive ROM machines. Although previous research compared the effectiveness of these interventions, no clinical differences were found in the overall outcomes for pain, function, or ROM between interventions or with a combination of interventions [5] [6].

Since pain is one of the primary complaints before TKA, it is commonly used by patients as a measure of the effectiveness of their surgery. However, research shows inconsistencies regarding the effectiveness of surgery and rehabilitation for lowering pain levels [7] [8]. Using a patient questionnaire to assess satisfaction, Baker et al. [7] found $82 \%$ of patients were satisfied with their pain level one year after surgery. The patients also reported pain was most common when walking long distances, kneeling, or shopping [7]. A similar study reported 44\% of patients still had persistent pain after surgery and $15 \%$ claimed to still have severe to extreme pain [8].

Like pain measurements, knee ROM measurements are also used to objectively measure the success of TKA rehabilitation. Although patients will have a large decrease in ROM immediately after surgery, most will regain their ROM within six months [2]. However, even with a full return to presurgical ROM val- 
ues, their outcomes are still not comparable to their healthy adult counterparts who do not have osteoarthritis or a history of TKA [9]. For instance, Bade et al. [2] reported average postoperative values for knee flexion ROM were $113.4^{\circ}$ at six months after TKA. This range is sufficient to complete most daily tasks, but patients would have difficulty with such tasks as getting into the bathtub or squatting, which both require about $125^{\circ}$ of knee flexion [2].

Perhaps the most important outcome measurement after TKA is the overall functional mobility of the patient. Pain and ROM both contribute to this outcome. After one year, Bade et al. [2] reported that postoperative TKA patients still had functional deficits when compared with healthy counterparts, specifically $18 \%$ slower walking speed, $51 \%$ slower stair climbing speed, and a nearly $40 \%$ deficit in quadriceps strength. Another study followed patients for an average of five years after TKA and found that overall satisfaction with their functional mobility level was $82 / 100$, but many reported difficulties performing advanced activities [10].

The GoKnee device was invented to expedite the rehabilitation process after TKA. The developers claim that ROM improvements can be made in half the typical rehabilitation time when the protocol is correctly followed. A key element of the device is the design, which facilitates ROM improvements through the orientation of the pads. These pads provide a mobilization at the knee joint, which theoretically allows the patient to mobilize the joint without a therapist present. The protocol is another key element of the device, and it uses the pad orientation to improve ROM through sustained mobilizations in combination with active movement into the end range. The protocol also improves ROM by incorporating motor learning principles. The push-pull portions of the protocol use progressive angular isometric loading and regressive angular isometric loading to improve the patient's usable ROM. If claims about how quickly the GoKnee improves ROM are accurate, the device has the potential to significantly reduce total healthcare expenditures after TKA.

Although the GoKnee is increasingly used in the clinical setting, to our knowledge, no studies have assessed its effect on treatment outcomes. The current literature reports gaps between the functional level of patients receiving traditional postoperative TKA rehabilitation and their healthy counterparts, and the effect of the GoKnee device on patient outcomes when incorporated into postoperative care is unclear. Therefore, the purpose of the current study was to investigate whether the GoKnee device significantly improved objective and functional measurements after TKA.

\section{Methods}

\subsection{Participants}

The current study used convenience sampling for participant recruitment, so the number of participants in the study was determined by the total number of available patients at the participating skilled nursing facilities or home health 
companies. Study inclusion criteria were any adult male or female patient who had recently undergone TKA and was currently receiving physical therapy services through a skilled nursing facility or home health.

\subsection{Instrumentation}

The GoKnee device was created by three physical therapists. It is constructed with polyvinyl chloride (PVC) piping and foam padding and has a breakaway feature to ensure patient safety. In the current study, the device was used while the participant was sitting upright in a standard chair with no armrests. The participant remained in the chair for all phases of the protocol. A standard long arm goniometer was used to perform all flexion and extension measurements.

\subsection{Study Protocol and Procedure}

The developers of the GoKnee established a research-based protocol that incorporates stretching, joint mobilizations, angular isometrics, and contract-relax techniques to maximize the effectiveness of the device. The protocol is divided into three phases of flexion, extension, and strengthening. Each participant of the current study followed a traditional TKA rehabilitation protocol and the GoKnee protocol exactly as designed. A complete protocol, including pictures, can be found in the Appendix.

During the flexion portion, the participant began with a two-minute warm-up that involved moving the device back and forth slowly and pushing further into the available ROM each time. Next, the knee was held in as much flexion as tolerable for two minutes. The protocol defines tolerable as being near a 7/10 rating on a subjective pain scale. The flexion protocol concluded with five continuous rounds of alternating pushing and pulling. For 20 seconds, the participant performed an isometric contraction of the quadriceps followed by full relaxation and then pulling of the knee into further flexion. This procedure was repeated for an additional four rounds.

The extension portion of the protocol did not have a warm-up since it immediately followed the flexion phase. The participant began by using the device to push the knee into as much extension as tolerable, about a 7/10 rating on a subjective pain scale, and holding this position for two minutes. This sustained stretch was followed with a 20 -second isometric contraction of the hamstring muscles, full relaxation, and then 20 seconds of lifting the lower leg and moving further into the newly available extension ROM. The participant was encouraged to move the device forward into the new range so that the hamstring contraction occurred isometrically. This cycle of hamstring and quadriceps contractions was repeated for an additional four cycles.

The protocol concluded with the quadriceps strengthening phase. For two minutes, the participant placed the knee over the top pad of the device, extended it, and held that position for at least five seconds. The participant then relaxed the leg into a flexed position for ten seconds and repeated the motion. With each 
repetition, the participant was encouraged to work further into the available extension ROM.

\subsection{Design and Data Collection}

The current study used a repeated measures design to specifically investigate the relationship between the independent variable of postoperative time frame and the dependent variables of pain, knee flexion, knee extension, TUG score, gait velocity, and 30-second sit-to-stand score. The physical therapists providing services collected data during the treatment sessions and included the inventors of the device. After discharge, the data were de-identified and given to study investigators for analysis. The data were categorized into four postoperative time frames for data analysis: days $2-6$, days $7-11$, days $12-16$, and 17+ days.

\subsection{Data Analysis}

All data were analyzed using Statistical Package for the Social Sciences software (SPSS) version 25. Mean and standard deviation (SD) were calculated for all postoperative time frame categories for each dependent variable. The mean was calculated as the sum of all of the data points in a category and then dived by the number of data points. The standard deviation was calculated as the square root of variance by determining each data point's deviation relative to the mean. A paired $t$ test was used to determine improvements over time by comparing means between postoperative time frames and dependent variables. Because of the number of possible combinations, a Bonferroni correction was used to decrease the risk of a type I error in reported results. A corrected $P$ of 0.008 was used for all comparisons.

\section{Results}

Twenty-nine participants completed the study, and their ages ranged from 50 90 years. Mean (SD) values for each dependent variable for all postoperative time frames are reported in Table 1. Differences were found for all variables

Table 1. Means (standard deviations) for functional and objective variables of the current study for postoperative time frame categories.

\begin{tabular}{ccccc}
\hline \multirow{2}{*}{ Variable } & \multicolumn{4}{c}{ Postoperative Days } \\
\cline { 2 - 4 } & $2-6$ & $7-11$ & $12-16$ & $17+$ \\
\hline Pain, 1-10 & $5.59(2.27)$ & $3.82(1.74)$ & $3.21(1.86)$ & $1.73(1.89)$ \\
Knee flexion, & $89.28(12.19)$ & $102.31(11.11)$ & $108.23(8.80)$ & $116.23(10.31)$ \\
Knee extension*, & $-3.45(4.99)$ & $-0.65(1.33)$ & $-0.10(1.33)$ & $0.77(1.88)$ \\
Timed Up and Go, s & $53.00(15.41)$ & $23.96(12.73)$ & $17.60(3.25)$ & $13.45(2.42)$ \\
Gait velocity m/s & $0.38(.30)$ & $0.64(.29)$ & $0.77(.12)$ & $0.81(.18)$ \\
30-Second sit-to-stand, reps & $2.36(2.48)$ & $7.08(3.45)$ & $7.87(2.15)$ & $9.91(2.12)$ \\
\hline
\end{tabular}

*Negative value indicates degrees lacking from full extension. 
Table 2. Comparisons between functional and objective variables of the current study and postoperative time frame categories to determine improvements over time.

\begin{tabular}{cccc}
\hline \multirow{2}{*}{ Variable } & \multicolumn{3}{c}{ Postoperative Time Frame Comparisons } \\
\cline { 2 - 4 } & $2-6$ vs $7-11$ & $7-11$ vs $12-16$ & $12-16$ vs 17+ \\
\hline Pain & $<0.001^{*}$ & $0.008^{*}$ & 0.12 \\
Knee flexion & $<0.001^{*}$ & $0.001^{*}$ & 0.01 \\
Knee extension & $0.001^{*}$ & $>0.99$ & $\mathrm{ND}$ \\
Timed Up and Go & $<0.001^{*}$ & 0.11 & 0.02 \\
Gait Velocity & $<0.001^{*}$ & 0.02 & 0.10 \\
30-Second sit-to-stand & $0.001^{*}$ & 0.56 & 0.03 \\
\hline
\end{tabular}

${ }^{*}$ Indicates significance per Bonferroni correction of $P<0.008 .{ }^{*} \mathrm{ND}$ : not enough data for this analysis.

when comparing postoperative days 2 - 6 and days $7-11$ (Table 2). Differences were found for pain $(P=0.008)$ and knee flexion $(P=0.001)$ when comparing postoperative days $7-11$ and days $12-16$. No other differences were found when comparing postoperative days $7-11$ and days $12-16$ (all $P>0.02$ ). No differences were found when comparing postoperative days $12-16$ and days $17+$ (all $P>0.01$ ).

\section{Discussion}

When supplementing traditional rehabilitation interventions with the GoKnee protocol in the current study, participants had statistically significant improvements in pain, knee flexion, knee extension TUG score, gait velocity, and 30-second sit-to-stand score. However, it is expected that functional and objective outcomes, such as those measured in the current study, will improve over time after TKA, regardless of the rehabilitation interventions patients received. Without control group receiving traditional rehabilitation interventions only, it is difficult to attribute our results strictly to the addition of the GoKnee protocol. Instead, our results suggest that the GoKnee device, when used with traditional exercises and interventions, subjectively and objectively improves patient outcomes.

Our findings that TUG scores and gait velocity improved after TKA were supported by existing research. A 2019 study by Iwata et al. [11] investigated the effect of quadriceps strength on the gait function of patients after TKA. The researchers measured gait velocity and TUG scores at two and three weeks after surgery [11]. Mean TUG scores were 14 seconds at two weeks and 12.7 seconds at three weeks [11]. We found similar TUG scores in our study: 17.60 seconds for postoperative days $12-16$ and 13.45 seconds for days 17+. Iwata et al. [11] reported mean gait velocity of $0.82 \mathrm{~m} / \mathrm{s}$ at two weeks and $0.89 \mathrm{~m} / \mathrm{s}$ at three weeks. After using the GoKnee device, our participants had mean gait velocity of $0.77 \mathrm{~m} / \mathrm{s}$ for postoperative days $12-16$ and $0.81 \mathrm{~m} / \mathrm{s}$ for days $17+$. This indicates that similar increases were seen in TUG scores and gait velocity post TKA in 
subjects who used the GoKnee and in those that did not.

In 2008, Lenssen et al. [12] investigated outcome measures of patients after TKA using a continuous passive motion machine. At postoperative day 17 , mean knee flexion values were $89.7^{\circ}$ in the control group and $93^{\circ}$ in the intervention group [12]. These reported values [12] are less than those of the current study; our mean knee flexion was $108^{\circ}$ degrees for postoperative days $12-16$. For knee extension ROM measurements, Lenssen et al. [12] reported lacking $5.7^{\circ}$ in the control group and $4.3^{\circ}$ of passive extension in the intervention group 17 days after surgery. Conversely, participants using the GoKnee protocol in our study reported a mean lacking of $0.1^{\circ}$ for postoperative days $12-16$. Thus, the GoKnee device had better outcomes in a shorter time than those reported by Lenssen et al. [12].

A 2010 study by Bade et al. [2] followed up with 24 patients one month after TKA. Mean active knee flexion ROM was $99.6^{\circ}$ and knee extension ROM was lacking $6.4^{\circ}$ [2]. In the current study, use of the GoKnee device resulted in improvements in both measures. At postoperative days $17+$, our mean knee flexion was $116.23^{\circ}$, and knee extension was $0.77^{\circ}$ of hyperextension. However, in the current study, the last day postoperative data was collected was day 24 , which is much earlier than the one month follow-up of Bade et al. [2]. Further, Bade et al. [2] also reported mean TUG scores of 14.6 seconds, which is slower than our 13.45 seconds for postoperative days $17+$.

The current study had several limitations. Most notably, we did not include a control group, and the inventors of the GoKnee device were involved with data collection. Another limitation was the lack of consistent postoperative day data collection. Specifically, we were unable to collect data on the same postoperative days for each participant. Because of natural variations in the amount of time available for interactions with patients from a skilled nursing facility or those receiving home health services, these differences in data collection were expected. One strength of the current study was that all our participants strictly adhered to the GoKnee protocol.

The data collection methods of the current study and the time frames assessed do not allow specific conclusions about the long-term benefits of the GoKnee device. Further, comparisons with previous studies are problematic since those studies mostly assessed patients one month to several years after TKA. For studies with a one-month follow-up, comparisons suggest that inclusion of the GoKnee protocol may result in significant improvements in functional and objective outcomes [2]. However, additional research is necessary to verify the efficacy of the device.

\section{Conclusions}

The prevalence of TKA is increasing yet there is not a consistent rehabilitation approach post-operatively. The GoKnee device was invented by physical therapists to improve objective and functional outcomes post-TKA. The purpose of 
this study was to investigate the accuracy of that goal. In addition to a traditional TKA rehabilitation program, participants also followed the GoKnee protocol, which addressed their knee flexion range of motion, knee extension range of motion, and quadriceps strengthening when they use the device.

The current study found that the GoKnee device significantly improved pain, knee flexion and extension ROM, and functional outcomes in the early phases of rehabilitation after TKA. Further, our ROM and gait velocity results were comparable to results from other studies. However, more research is needed to evaluate the efficacy of the GoKnee device. For instance, studies should include a control group receiving traditional rehabilitation interventions only. Studies with longer follow-up periods or a randomized control trial should also be conducted to assess the long-term benefits of the GoKnee device and determine whether its use provides improved objective and functional outcomes for patients receiving rehabilitation services after TKA.

\section{Acknowledgements}

We would like to thank the creators of the GoKnee device, Shehla Rooney, PT, Sonya Briggs, PT, and Nikki Orazine, DPT, for their support throughout this work.

We are grateful to Deb Goggin, Research Support and Scientific Writer at A.T. Still University for her support on this work.

\section{Conflicts of Interest}

The authors declare no conflicts of interest.

\section{References}

[1] Kurtz, S., Ong, K., Lau, E., Mowat, F. and Halpern, M. (2007) Projections of Primary and Revision Hip and Knee Arthroplasty in the United States from 2005 to 2030. The Journal of Bone and Joint Surgery, 89, 780-785. https://doi.org/10.2106/JBJS.F.00222

[2] Bade, M.J., Kohrt, W.M. and Stevens-Lapsley, J.E. (2010) Outcomes Before and After Total Knee Arthroplasty Compared to Healthy Adults. The Journal of Orthopaedic and Sports Physical Therapy, 40, 559-567. https://doi.org/10.2519/jospt.2010.3317

[3] Kennedy, D.M., Stratford, P.W., Riddle, D.L., Hanna, S.E. and Gollish, J.D. (2008) Assessing Recovery and Establishing Prognosis Following Total Knee Arthroplasty. Physical Therapy, 88, 22-32. https://doi.org/10.2522/ptj.20070051

[4] Nuzzi, E.M. (2008, November 26) Standard of Care: Total Knee Arthroplasty. Brigham and Women's Hospital Department of Rehabilitation Services. https://www.brighamandwomens.org/assets/BWH/patients-and-families/rehabilitat ion-services/pdfs/knee-total-knee-arthroplasty-bwh.pdf

[5] Beaupré, L.A., Davies, D.M., Jones, C.A. and Cinats, J.G. (2001) Exercise Combined With Continuous Passive Motion or Slider Board Therapy Compared With Exercise Only: A Randomized Controlled Trial of Patients Following Total Knee Arthroplasty. Physical Therapy, 81, 1029-1037. https://doi.org/10.1093/ptj/81.4.1029 
[6] Worland, R.L., Arredondo, J., Angles, F., Lopez-Jimenez, F. and Jessup, D.E. (1998) Home Continuous Passive Motion Machine versus Professional Physical Therapy Following Total Knee Replacement. The Journal of Arthroplasty, 13, 784-787. https://doi.org/10.1016/s0883-5403(98)90031-6

[7] Baker, P.N., van der Meulen, J.H., Lewsey, J. and Gregg, P.J. (2007) The Role of Pain and Function in Determining Patient Satisfaction after Total Knee Replacement: Data from the National Joint Registry for England and Wales. The Journal of Bone and Joint Surgery, 89-B, 893-900.

https://doi.org/10.1302/0301-620x.89b7.19091

[8] Wylde, V., Hewlett, S., Learmonth, I.D. and Dieppe, P. (2011) Persistent Pain after Joint Replacement: Prevalence, Sensory Qualities, and Postoperative Determinants. Pain, 152, 566-572. https://doi.org/10.1016/j.pain.2010.11.023

[9] Yoshida, Y., Mizner, R.L., Ramsey, D.K. and Snyder-Mackler, L. (2008) Examining Outcomes From Total Knee Arthroplasty and the Relationship Between Quadriceps Strength and Knee Function Over Time. Clinical Biomechanics, 23, 320-328. https://doi.org/10.1016/j.clinbiomech.2007.10.008

[10] Matsuda, S., Kawahara, S., Okazaki, K., Tashiro, Y. and Iwamoto, Y. (2013) Postoperative Alignment and ROM Affect Patient Satisfaction After TKA. Clinical Orthopaedics and Related Research, 471, 127-133. https://doi.org/10.1007/s11999-012-2533-y

[11] Iwata, A., Sano, Y., Wanaka, H., Yamamoto, S., Yano, Y. and Iwata, H. (2019) Different Improvement Trends in Gait Function and Quadriceps Strength Early After Total Knee Arthroplasty. Journal of Physical Therapy Science, 31, 57-62. https://doi.org/10.1589/jpts.31.57

[12] Lenssen, T.A.F., van Steyn, M.J.A., Crijns, Y.H.F., Waltjé, E.M.H., Roox, G.M., Geesink, R.J.T, van den Brandt, P.A. and De Bie, R.A. (2008) Effectiveness of Prolonged Use of Continuous Passive Motion (CPM), as an Adjunct to Physiotherapy, after Total Knee Arthroplasty. BMC Musculoskeletal Disorders, 9, Article No. 60. https://doi.org/10.1186/1471-2474-9-60 


\section{Appendix. GoKnee Protocol}

\section{GoKnee Patient Protocol}

\section{Purpose}

The GoKnee device is used to increase movement at the knee joint and increase strength in the quad (thigh) muscles.

\section{Part 1: Range of Motion Protocol}

\section{Positioning for Knee Bending}

- Begin in a seated position.

- Place the device on the floor with the handles facing the ceiling. (1a)

- Insert foot between the two pads. (1b)

- Lift the device by the handles until the knee is resting on the top pad and your ankle is behind the bottom pad. (1c)

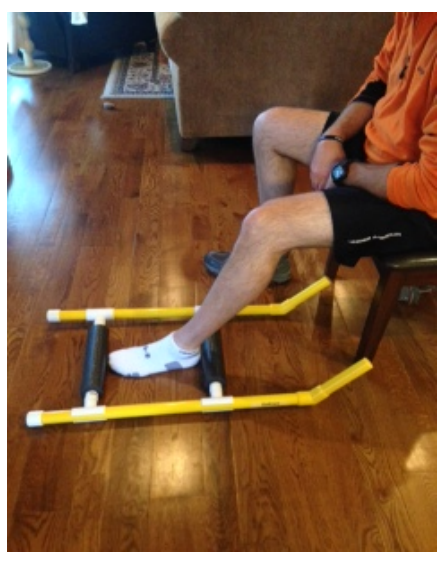

1a-device on the floor

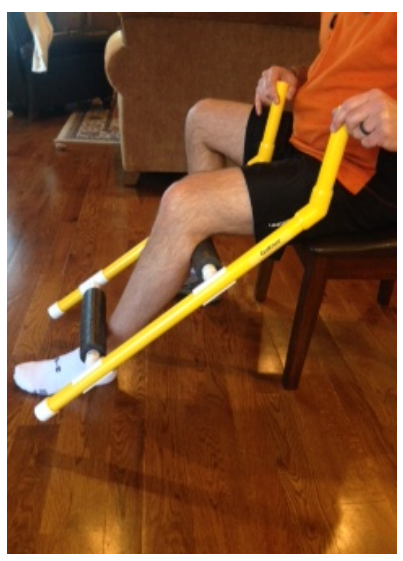

1b-foot between the pads

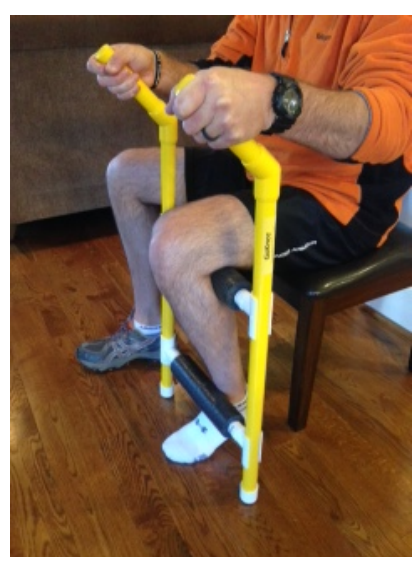

1c-device is upright and ankle behind pad

In the bending position, described above, perform the following exercises.

- For 2 minutes, move the device back and forth slowly by evenly pushing and pulling on the handles, stretching your knee a little further each time.

- For 2 minutes, hold your knee bent as far as you can. This hold should be at a 7/10 pain level or moderate discomfort.

Follow with 5 continuous rounds of alternating push and pull.

- For 20 seconds, push into the bottom pad with your ankle, as if you are trying to straighten your leg, but do not allow the GoKnee device to move.

- For 20 seconds, immediately pull your knee back as far as you can, using the GoKnee device to assist with further bending. This motion should be a further bend each time.

\section{${ }^{*}$ After the 5 rounds of this activity slowly let your knee straighten and remove it from the GoKnee device}

\section{Positioning for Knee Straightening}

- Hold the device completely upright and hold onto the handles. (2a)

- Insert your foot between the pads and rest your ankle on the bottom pad. (2b, 2c) 
- Place the device, with your leg resting on the bottom pad, on a coffee table, ottoman, or stool. (2d, 2e)

- Slide the adjustable top pad to just above your knee joint. (2f)

\section{Floor Position for Straightening}

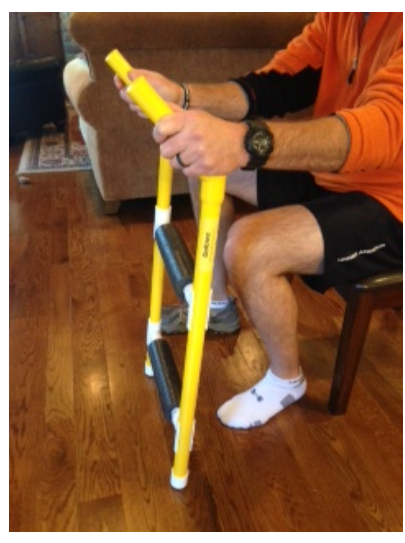

2a-device is upright

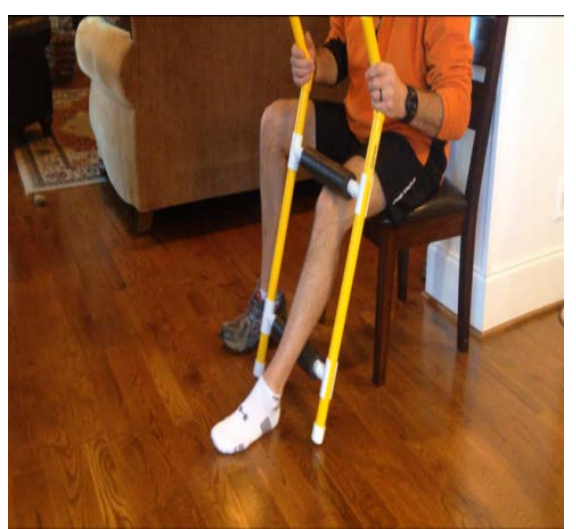

2b-foot between pads

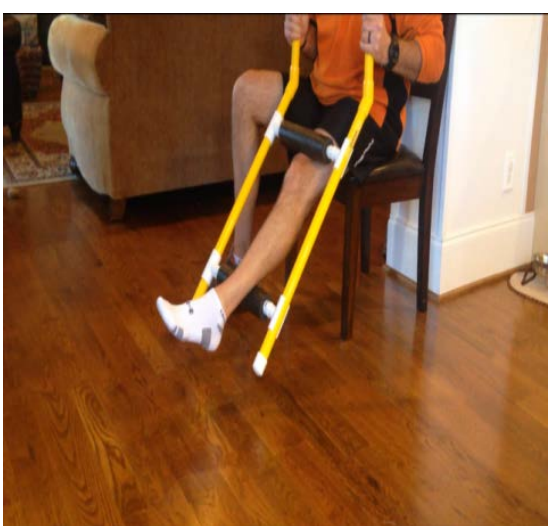

2c-ankle resting on bottom pad

\section{Propped Position for Straightening}

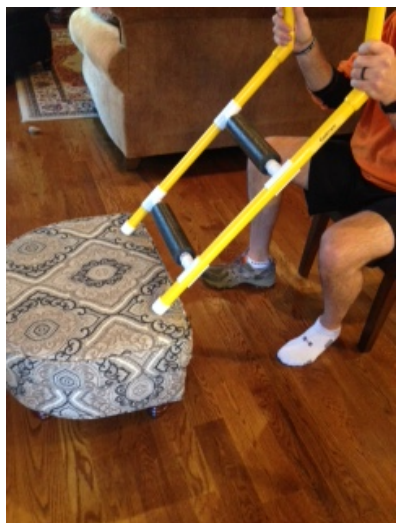

2d-place device on stool

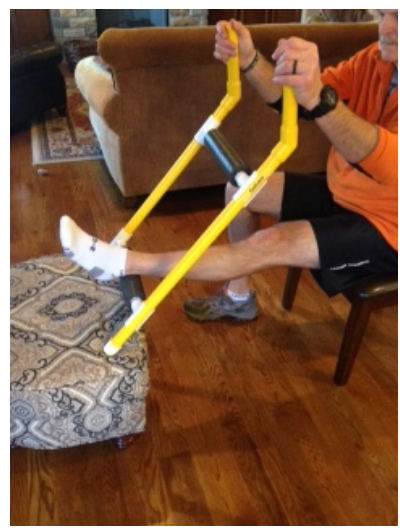

2e-foot resting on bottom pad

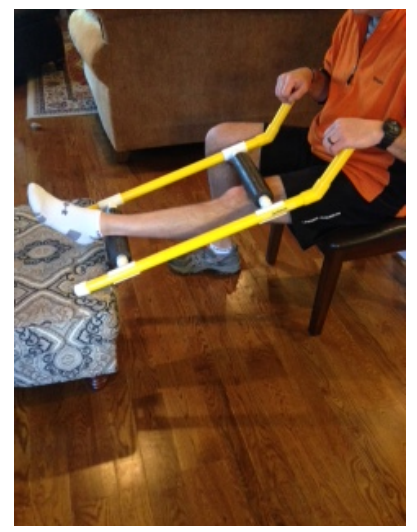

2f-top pad is above knee joint

In the straightening position, described above, perform the following exercises.

- For 2 minutes, push your knee in a straight position with an even pressure on the handles from your hands. This motion should be at a 7/10 pain level or moderate discomfort.

Follow with 5 continuous rounds of alternating push and pull.

- For 20 seconds, pull your foot into the bottom pad as hard as you can without letting the GoKnee device move.

- For 20 seconds, lift your foot off the ankle pad while pushing your hands down to add pressure to your knee.

*After 5 rounds of this activity remove your leg from the GoKnee device and relax 


\section{Part 2: Strengthening Protocol}

\section{Positioning for Quadriceps Strengthening:}

- Begin in a seated position. (3a)

- Place the GoKnee device completely upright. (3a)

- Rest your knee on the top pad as your foot relaxes into a bending position. (3a)

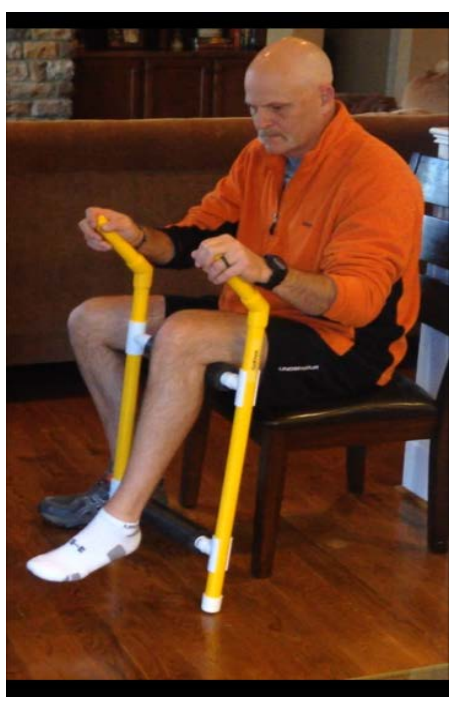

3a-seated, device upright, knee on top

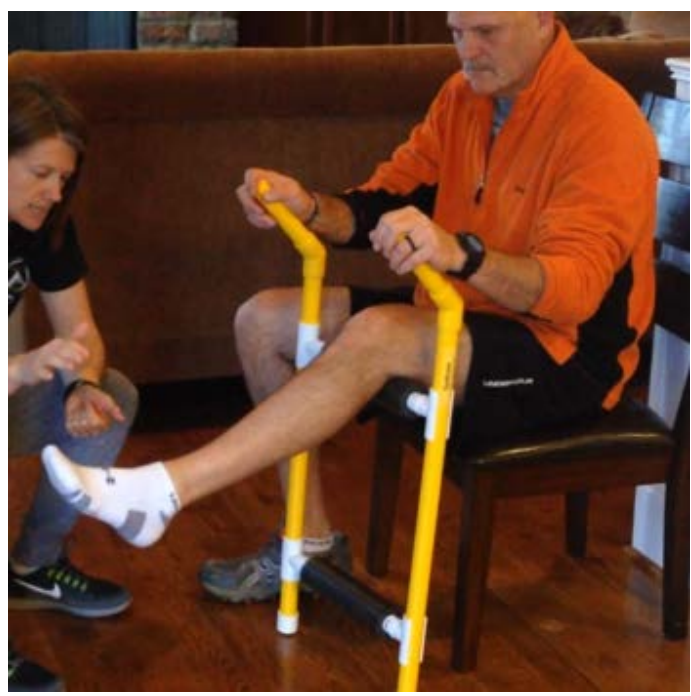

3b-perform leg kicks/knee extension

In the strengthening position, described above, perform the following exercise.

- Perform leg kicks by bringing your foot upward and holding it for at least 5 seconds. (3b)

- Rest your knee in a bent position for 10 seconds.

- Repeat this sequence for 2 minutes.

*Each time you kick, your foot you should be trying to kick higher while keeping the back of your knee on the top pad

\section{Schedule of the Routine}

- Perform this routine in the order specified above.

- Perform this routine a minimum of 3 times a day.

- Perform this routine a maximum of 6 times a day.

- Apply an ice pack to the knee for 15 minutes after each time the routine is performed.

- If you have increased swelling, significant redness, or lasting pain greater than an 8/10 pain level at rest, please call your physical therapist at: 